\title{
Is policy 'liberalization' associated with higher odds of adolescent cannabis use? A re-analysis of data from 38 countries.
}

\author{
Alex Stevens, School of Social Policy, Sociology and Social Research, University of Kent, \\ Medway, United Kingdom \\ Email: a.w.stevens@kent.ac.uk
}

This is the pre-print submitted manuscript of an article which was accepted for publication as a commentary in the International Journal of Drug Policy in January 2019

\begin{abstract}
Background: Policy makers worldwide face the choice of whether to reform cannabis policy from the 'full prohibition' model. A paper by Shi, Lenzi and An (2015) suggested that such 'liberalization' is significantly associated with higher odds of adolescent cannabis use.

Aim: To test the validity and reliability of Shi et al's conclusion that the HBSC data show an association between policy 'liberalization' and increased likelihood of adolescent cannabis use.

Methods: Replication and re-analysis of the same pooled data from three waves of the Health Behaviour in School-aged Children (HBSC) survey (2001/2, 2005/6 and 2009/10). This replicates - as far as possible - the coding and analytical strategy of the Shi et al article. The re-analysis makes some improvements by: excluding a variable ('number of siblings') for which many cases have missing data; including available data from the theoretically relevant case of Sweden for the latter two waves of the HBSC survey, which Shi et al omit; and including random slopes for gender between countries as well as random intercepts for countries in the mixed effects model, as the predictive effect of gender on cannabis use varies across countries. Results: Shi et al's verbal summary of their findings is not supported by detailed interpretation of their own numerical results. Without making the suggested amendments, it is possible to find a statistically significant association between policy 'liberalization' and higher odds of some measures of adolescent cannabis use. But when these improvements are made, this association becomes statistically nonsignificant.

Conclusion: Using a larger and more theoretically relevant sample of the HBSC respondents and an improved statistical model shows that the HBSC data do not reveal a statistically significant association between policy 'liberalization' and higher odds of adolescent cannabis use.
\end{abstract}

\section{Keywords}

Cannabis; policy; youth; adolescence; HBSC; multilevel regression; replication; open science 


\section{Introduction}

In several parts of the world, a 'kind of peace' is breaking out in the 'war on drugs' (McLean, 2018). Countries including Canada, Uruguay, Jamaica, Portugal, the Czech Republic, Spain, Germany, Norway, Italy and several US states have reformed laws on cannabis. However, little is known about the population-level effects of such legal changes. The research that has been done has tended to suggest mixed results (Babor et al., 2018; Waddell \& Wilson, 2017).

One study that is increasingly cited in this discussion is by Shi et al (Shi, Lenzi, \& An, 2015). It presented multilevel logistic regression analysis of data from the Health Behaviour in School-aged Children: WHO Collaborative Cross-National survey/study (HBSC). It took into account several variables, including a dichotomous indicator of policy 'liberalization'. It concluded that there is a statistically significant association between policy 'liberalization' and an increased likelihood of adolescent cannabis use.

The Shi et al article has been used by policy advocates in Ireland, for example, to resist calls for decriminalisation of cannabis (Lawler, 2016, personal communication). It has also been cited by several researchers as evidence of an association between national policy and prevalence of cannabis use (Burdzovic Andreas \& BrettevilleJensen, 2017; Chapman et al., 2017; Crowley, Collins, Delargy, Laird, \& Van Hout, 2017; Dirisu, Shickle, \& Elsey, 2016; Mark, Gryczynski, Axenfeld, Schwartz, \& Terplan, 2017; Murray, Quigley, Quattrone, Englund, \& Di Forti, 2016; Pacini, Maremmani, Patricio, Barra, \& Maremmani, 2017; Peltzer \& Pengpid, 2017; Turan Sonmez, Kilicasla, \& Akbas, 2017; Waddell \& Wilson, 2017; Weinberger et al., 2018). So Shi et al's findings are of high academic and policy interest.

Across the social sciences, the need for 'open science' is becoming increasingly clear (Open Science Collaboration, 2015; Silberzahn \& Uhlmann, 2015), including in the field of drug policy (Wall et al., 2016). Replication and re-analysis are crucial for 'opening up' the study of drug use and policy (Munafò, 2016; Munksgaard, Demant, \& Branwen, 2016). The data on which Shi et al's findings are based are available for secondary analysis for not-for-profit purposes by other researchers (Roberts et al., 
2009). This means it is possible to attempt to replicate this analysis and - if found necessary - to improve upon its approach to testing the hypothesis that there is an association between a country's policy type and the odds of adolescent cannabis use in that country.

This article will report such a replication and re-analysis. It will summarise the data and methods, the coding and analytical approach adopted by Shi et al, and will question their verbal interpretation of their own numerical findings. It will then report on the replication and re-analysis that was carried out. The aim of this article is not to provide an original analysis of the HBSC data. Rather, it is to test whether the conclusions given by Shi et al are robust.

\section{Data and methods}

The findings reported here are based on secondary analysis of pooled data from three waves of the HBSC survey, collected in 2001/2, 2005/6 and 2009/10 across 38 countries (HBSC Network, 2014; Roberts et al., 2009). The data come from adolescents (average age 15) in randomly surveyed schools. They are asked to complete a standardised, pen-and-paper questionnaire with comparable questions across the waves and countries of the survey. As Shi et al report, the aim is to create estimates of national prevalence of cannabis use that are accurate to within three percentage points. The HBSC survey received ethical approval for participating institutions in the 38 countries covered.

In order to analyse whether there is an association between policy type and cannabis use, Shi et al categorise countries into policy types. They do this by adopting the four policy types that Room et al (2008) describe, with supplementary information from reports published by the European Monitoring Centre for Drugs and Drug Addiction (EMCDDA). Room et al's four categories are 'full prohibition', 'depenalization', 'decriminalization' and 'partial prohibition'.

The country coding decisions for countries are reported indirectly by Shi et al; they provide a list of which countries changed their policies from full prohibition and when, 
rather than reporting exactly how each country was coded for each survey year. Table 1 reproduces Shi et al's list of countries with their verbal description of their policies. It also shows directly how these countries were coded for the replication and re-analysis in this article. See Røgeberg \& Stevens (2016) for a discussion of problems in this coding of countries.

Shi et al present results from two-level logistic regression models. The dependent variables are whether the respondent reported using cannabis ever, in the past year or 'regularly' (defined as 40 times or more in their lifetime). The HBSC also collects data on individual characteristics, including: whether the respondent lives with both parents, their number of siblings and friends, time spent with friends, difficulties in communication with parents and friends, family affluence and psychological complaints. These are entered as fixed effects in the models. Shi et al also enter as fixed effects the respondent's country's policy type and level of gross domestic product (GDP) per capita, as well as a dummy variable indicating to which wave of the HBSC survey the respondent belonged. Random intercepts (but not random slopes) for countries are entered in the model as random effects. Shi et al run models for the cannabis use variables by entering a variable for all four types (comparing odds of use for respondents in 'depenalization', 'decriminalization', and 'partial prohibition' countries to those in 'full prohibition countries') and by comparing these odds between respondents in countries that have any 'liberalization' and those in 'full prohibition' countries. This article will focus on the results for any 'liberalization'. This is for two reasons. One is the difficulty of accurately assigning countries to policy types (Røgeberg \& Stevens, 2016). The other is that this relationship between adolescent cannabis use and 'liberalization' in general has been the main finding cited from the Shi et al article by other researchers.

With any given dataset, it is possible to apply different analytical models, which may produce different results (Humphreys, de la Sierra, \& van der Windt, 2013). This makes it very important that the models chosen provide the best possible test of the hypothesis. These issues were addressed in designing the re-analysis of the HBSC data, which is described below. Indicators of goodness of fit are used to test which models best fit these particular data. It should be remembered that other data and other analyses might produce different findings. 
Table 1: Coding of countries by policy

type

\begin{tabular}{|c|c|c|c|c|}
\hline \multirow[t]{2}{*}{ Country } & \multirow[t]{2}{*}{ Shi et al description (table 2) } & \multicolumn{3}{|c|}{ Coding for replication and re-analysis } \\
\hline & & $2001 / 2$ & $2005 / 6$ & $2009 / 10$ \\
\hline Austria & Partial Prohibition, 1998 & 1 & 1 & 1 \\
\hline Armenia & Decriminalization, 2008 & 0 & 0 & 1 \\
\hline Belgium & Decriminalization, 2003 & 0 & 1 & 1 \\
\hline Bulgaria & Full Prohibition & 0 & 0 & 0 \\
\hline Canada & Depenalization, 1996 \& Partial Prohibition, 2001 & 1 & 1 & 1 \\
\hline Croatia & Full Prohibition & 0 & 0 & 0 \\
\hline Czech Repu & Decriminalization, 2010 & 0 & 0 & 1 \\
\hline Denmark & Decriminalization, 2004 & 0 & 1 & 1 \\
\hline Estonia & Decriminalization, 2002 & 0 & 1 & 1 \\
\hline Finland & Full Prohibition & 0 & 0 & 0 \\
\hline France & Depenalization, 1999 & 1 & 1 & 1 \\
\hline Germany & Partial Prohibition, 1994 & 1 & 1 & 1 \\
\hline Greece & Full Prohibition & 0 & 0 & 0 \\
\hline Greenland & Full Prohibition & 0 & 0 & 0 \\
\hline Hungary & Full Prohibition & 0 & 0 & 0 \\
\hline Iceland & Full Prohibition & 0 & 0 & 0 \\
\hline Ireland & Full Prohibition & 0 & 0 & 0 \\
\hline Israel & Partial Prohibition, 1992 & 1 & 1 & 1 \\
\hline Italy & Decriminalization, 1990 & 1 & 1 & 1 \\
\hline Latvia & Decriminalization, 1999 & 1 & 1 & 1 \\
\hline Lithuania & Decriminalization, 1998 (ended 2003) & 1 & 0 & 0 \\
\hline Luxembour & Decriminalization, 2001 & 1 & 1 & 1 \\
\hline Malta & Full Prohibition & 0 & 0 & 0 \\
\hline Netherland: & Partial Prohibition, 1976 & 1 & 1 & 1 \\
\hline Norway & Full Prohibition & 0 & 0 & 0 \\
\hline Poland & Full Prohibition & 0 & 0 & 0 \\
\hline Portugal & Decriminalization, 2001 & 1 & 1 & 1 \\
\hline Romania & Full Prohibition & 0 & 0 & 0 \\
\hline Russia & Decriminalization, 2004 & 0 & 1 & 1 \\
\hline Slovakia & Full Prohibition & 0 & 0 & 0 \\
\hline Slovenia & Full Prohibition & 0 & 0 & 0 \\
\hline Spain & Partial Prohibition, 1992 & 1 & 1 & 1 \\
\hline Sweden & Full Prohibition & 0 & 0 & 0 \\
\hline Switzerland & Full Prohibition & 0 & 0 & 0 \\
\hline Ukraine & Full Prohibition & 0 & 0 & 0 \\
\hline Macedonia & Full Prohibition & 0 & 0 & 0 \\
\hline UK & Depenalization, 2004 (ended 2009) & 0 & 1 & 0 \\
\hline USA & Partial prohibition in some jurisdictions, 1996 & 1 & 1 & 1 \\
\hline & & Key: 0 = 'Fu & ohibition' & \\
\hline & & 1 = 'Liberal & & \\
\hline
\end{tabular}




\section{Shi et al's analysis}

The results of Shi et al's models for the odds ratios of cannabis use (lifetime, past year and 'regular') are reproduced in Table 2. As these are odds ratios, a difference from one suggests that that variable may have a predictive effect on the odds of cannabis use.

Table 2: Multilevel logistic regressions for adolescent cannabis use. HBSC 2001-2010 (N=172,894). Results (odds ratios) reported by Shi et al 2015 (table 3)

\begin{tabular}{|l|l|l|l|}
\hline Individual-level variables & Lifetime use & Past year use & Regular use \\
\hline Boy & $1.91^{* * *}$ & $1.86^{* * *}$ & $3.08^{* * *}$ \\
\hline Living with both parents & $0.63^{* * *}$ & $0.63^{* * *}$ & $0.55^{* * *}$ \\
\hline Number of siblings & 1.00 & 0.99 & $1.04^{* * *}$ \\
\hline Difficulty of communication with parents & $1.34^{* * *}$ & $1.36^{* * *}$ & $1.30^{* * *}$ \\
\hline Difficulty of communication with friends & $0.72^{* * *}$ & $0.72^{* * *}$ & $0.69^{* * *}$ \\
\hline Number of friends & $1.04^{* * *}$ & $1.03^{* * *}$ & 1.00 \\
\hline Time spent with friends & $1.41^{* * *}$ & $1.41^{* * *}$ & $1.55^{* * *}$ \\
\hline Psychological complaints & $1.48^{* * *}$ & $1.51^{* * *}$ & $1.63^{* * *}$ \\
\hline Family affluence $=$ medium & $1.10^{* * *}$ & $1.11^{* * *}$ & 0.96 \\
\hline Family affluence $=$ high & $1.17^{* * *}$ & $1.22^{* * *}$ & 0.96 \\
\hline Country-level variables & & & \\
\hline Cannabis liberalization & $1.10^{* * *}$ & $1.09^{* *}$ & $1.23^{* *}$ \\
\hline Cannabis liberalization*boy & $0.80^{* * *}$ & $0.82^{* * *}$ & $0.77^{* * *}$ \\
\hline Per-capita GDP level $=$ second tertile & $1.17^{*}$ & 1.03 & $1.59^{* *}$ \\
\hline Per-capita GDP level $=$ third tertile & $1.38^{* * *}$ & 1.12 & $1.73^{* * *}$ \\
\hline Survey $=2005 / 6$ & $0.76^{* * *}$ & $0.69^{* * *}$ & $0.70^{* * *}$ \\
\hline Survey $=2009 / 10$ & $0.74^{* * *}$ & $0.68^{* * *}$ & $0.62^{* * *}$ \\
\hline${ }^{*}$ p $<0.05,{ }^{* *} p<0.01,{ }^{* *} p<0.001$ & & & \\
\hline & & & \\
\hline
\end{tabular}

Shi et al include the interaction between gender (i.e. being a boy, as boys are coded a 1 and girls as 0 in the dataset) and the policy type in their models. For girls in a 'liberalized' country, the odds ratio for lifetime cannabis use relative to girls in a 'full prohibition' country is simply the main effect (1.1). For boys in 'liberalized' countries, the odds ratio relative to boys in 'full prohibition' countries is calculated by multiplying the main effect by the interaction effect $(1.1 \times 0.8=0.88)$. Note that the resulting odds ratio for boys in 'liberalized' countries is below one, which implies that boys in 'liberalized' countries have lower odds of cannabis use than boys in 'full prohibition' countries when the predictive influence of all the other variables in the model is taken 
into account. Shi et al do not include the implied odds ratio for boys in 'liberalized' countries in their table of results, but it has been included here as the bottom, italicised row of Table 2.

Shi et al's verbal interpretation of these numerical results is stated as, '[o]verall, cannabis liberalization was associated with higher likelihood of ever use, past-year use, and regular use of cannabis'. They also report, 'the associations between cannabis use and cannabis control policies were in general smaller in boys'.

\section{Issues in interpretation}

This verbal interpretation of the numerical model results is questionable. As shown above, the models' implied odds ratios for boys in 'liberalized' countries are below one, suggesting that there may be contradictory associations between policy type and odds of cannabis use between genders. Shi et al report that this association is weaker for boys, but do not verbally state the finding from their model that the association for boys is in the opposite direction than for girls. They do not report the marginal effect of policy 'liberalization' across both genders, but it is possible to impute this effect. This calculation produces odds ratios across both genders of 0.96 for lifetime use, 0.96 for past year use, and 1.02 for 'regular' use for adolescents living in 'liberalized' countries relative to those in 'full prohibition' countries (see Røgeberg \& Stevens, 2016 for the full calculation). It is not possible to test the statistical significance of these imputed odds ratios, given the parameters reported by She et al. But they do not suggest a consistent effect of 'liberalization' in increasing the 'likelihood' of cannabis use. The verbal summary that Shi et al give of their findings is therefore not a valid interpretation of their own numerical results. 


\section{Replication of Shi et al's analysis}

In order to test whether the numeric results reported by Shi et al are robust, the raw HBSC data for the variables that they use were subjected to a coding strategy which was - as far as possible - the same as reported by Shi et al. ${ }^{1}$

The data were then analysed (using the weighting variable included in the HBSC dataset, as Shi et al also report doing) in generalised linear mixed effects logistic regression models with random intercepts for countries. Shi et al report using Stata 12 to run their analysis. This replication and re-analysis reported here was carried out using the Ime4 package in the R open source statistical software environment (Bates, Mächler, Bolker, \& Walker, 2015). ${ }^{2}$

\footnotetext{
${ }^{1}$ The only exception to this was for Shi et al's derived variables 'difficulty of communication with parents' and 'difficulty of communication with friends'. These were based on questions on how easy the respondent finds it to talk about important matters with their mother and father, and with best friends, friends of the same sex and friends of the opposite sex. Shi et al report that the responses are in four categories, from very easy (1) to very difficult (4), but there is actually a fifth category (5) for 'don't have or don't see' parents or friends. It was not obvious how Shi et al dealt with such responses. For the replication and re-analysis, these scores were reversed in order to create new, derived variables for 'family communication' and 'friend communication', with people who reported not having or not seeing parents or friends therefore rated as having zero communication. Data for the countries' levels of GDP per capita were taken from Shi et al's Table 2.
}

2 The model equation of the average fitted regression line, expressing the relationship between the log-odds of reporting cannabis use and the other variables in the model is:

$$
\begin{aligned}
& \log \left(\frac{P_{i j}}{1-P_{i j}}\right)=\beta_{0}+\beta_{1} \text { BothParHome }_{i j}+\beta_{2} \text { NumSib }_{i j}+\beta_{3} \text { NumFriends }_{i j}+\beta_{4} \text { FamSup }_{i j}
\end{aligned}
$$

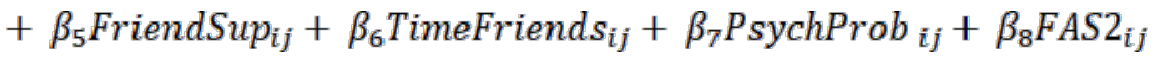

$$
\begin{aligned}
& +\beta_{9} F_{A S 3_{i j}}+\beta_{10} \text { PolLib }_{i j}+\beta_{11} \text { PolLib } * \text { Boy }_{i j}+\beta_{12} G D P 2_{i j}+\beta_{13} G D P 3_{i j} \\
& +\beta_{14} \text { HBSC2006 }_{i j}+\beta_{15} \text { HBSC2010 }_{i j}+\beta_{16} \text { Gender }_{i j}+u_{0 j}+\varepsilon_{i j}
\end{aligned}
$$

In this equation, $\mathrm{P}$ represents the probability of a respondent $i$ in country $j$ reporting any cannabis use in the stated period; lifetime, past year or 'regular'. 'BothParHome' is a dichotomous variable indicating whether the respondent lives with both parents. 'NumSib' is a derived variable calculated by adding the number of sisters to the number of brothers reported by the respondent. 'NumFriends' is the number of friends reported by the respondent. 'FamSup' is the derived variable described above for communication with family. 'FriendSup' is the derived variable described above for communication with friends. 'TimeFriends' represents the average number of days per week the respondent reported spending time with friends after school and in the evening. 'PsychProb' represents the average response to questions on a five point scale which was worded: 'in the last 6 months: how often have you had the following: 1) feeling low, 2) irritability or bad temper; 3) feeling nervous; 4) difficulties in getting to sleep; and 5) feeling dizzy'. Higher values represent reporting more frequent psychological problems. 'FAS2' is a dichotomous variable for whether the respondent reported their family as rating 
The results are shown in Table 3. For ease of comparison with Shi et al's

presentation of their results, this table shows only the odds ratios for the fixed effects

in the models. Full results are shown in Supplementary Table $1 .{ }^{3}$

Table 3: Multilevel logistic regressions for adolescent cannabis use. Results (odds ratios) of replication of Shi et al 2015 ( $N=115,504)$.

\begin{tabular}{|c|c|c|c|c|c|c|}
\hline \multirow[b]{2}{*}{ Individual-level variables } & \multicolumn{2}{|c|}{ Lifetime use } & \multicolumn{2}{|c|}{ Past year use } & \multicolumn{2}{|l|}{ Regular use } \\
\hline & Odds ratio & Sig' & Odds ratio & Sig' & Odds ratio & $\underline{\text { Sig' }}$ \\
\hline Boy & 2.00 & $* * *$ & 1.94 & $* * *$ & 3.04 & $* * *$ \\
\hline Living with both parents & 0.66 & $* * *$ & 0.69 & $* * *$ & 0.63 & $* * *$ \\
\hline Number of siblings & 1.00 & & 1.00 & & 1.03 & $* *$ \\
\hline Family communication & 0.76 & $* * *$ & 0.75 & $* * *$ & 0.75 & $* * *$ \\
\hline Friend communication & 1.43 & $* * *$ & 1.43 & $* * *$ & 1.27 & $* * *$ \\
\hline Number of friends & 1.02 & $*$ & 1.01 & & 0.99 & \\
\hline Time spent with friends & 1.43 & $* * *$ & 1.42 & $* * *$ & 1.59 & $* * *$ \\
\hline Psychological complaints & 1.50 & $* * *$ & 1.51 & $* * *$ & 1.63 & $* * *$ \\
\hline Family affluence $=$ medium & 1.12 & $* * *$ & 1.14 & $* * *$ & 1.00 & \\
\hline Family affluence $=$ high & 1.22 & $* * *$ & 1.25 & $* * *$ & 1.00 & \\
\hline \multicolumn{7}{|l|}{ Country-level variables } \\
\hline Cannabis liberalization & 1.33 & $* * *$ & 1.24 & $* * *$ & 1.29 & $* *$ \\
\hline Cannabis liberalization*boy & 0.78 & $* * *$ & 0.82 & $* * *$ & 0.79 & $* *$ \\
\hline Per-capita GDP level = second tertile & 1.31 & & 1.46 & & 2.69 & $* *$ \\
\hline Per-capita GDP level = third tertile & 1.64 & & 1.83 & $*$ & 3.84 & $* * *$ \\
\hline Survey $=2005 / 6$ & 0.76 & $* * *$ & 0.68 & $* * *$ & 0.69 & $* * *$ \\
\hline Survey $=2009 / 10$ & 0.70 & $* * *$ & 0.65 & $* * *$ & 0.58 & $* * *$ \\
\hline$* \mathrm{p}<0.05, * * \mathrm{p}<0.01, * * * \mathrm{p}<0.001$ & & & & & & \\
\hline
\end{tabular}

4-5 on the Family Affluence Scale. 'FAS3' is a dichotomous variable for whether the respondent reported their family as rating 6-9 on this scale. 'PolLib' is the variable for whether the respondent lived in a country that was coded (as in Table 1) as having a 'liberalized' cannabis policy. 'PolLib*Boy' is the interaction between the policy variable and the gender variable. 'GDP2' is a dichotomous variable indicating whether the respondent lived in a country in the middle tertile of GDP per head. 'GDP3' is a dichotomous variable for whether the respondent lived in a country in the highest tertile for GDP per head. The two HBSC variables are dummy variables for the year of the survey to which the respondent responded. 'Gender' denotes whether the respondent is a girl or a boy (with boys coded as 1 and girls as 0 ). The intercept $\beta_{0}$ is shared by all countries, while the random effect $u_{0 j}$ is specific to country $j$. The symbol $\varepsilon$ represents the observation-specific error. The $\mathrm{R}$ codes for these models was glmer([cannabis variable] Gender + BothParHome + NumSib + FamSup + FriendSup + NumFriend + TimeFriends + PsychProb + FAS2 + FAS3 + PolLib + PolLibBoy + GDPS2 + GDPS3 + HBSC2006 + HBSC2010 + (1 | Country), family = binomial("logit"), data $=[$ the dataset $]$, weight $=$ Weight $)$.

${ }^{3}$ Shi et al do not report what approach they used for approximation. The Ime4 package in $\mathrm{R}$ uses Laplace approximation (with 1 integration point) as its default, which was used for the models reported in Tables 3 and 4. The default in Stata is to use a 7 point quadrature fit. In order to check whether the results reported here were sensitive to the approximation, the models were also run with a 7 point quadrature fit in Ime4. This produced slight changes to the fixed effects reported in Table 3 , but these were usually not noticeable within two decimal points for these odds ratios. 
Two main differences stand out from the comparison of this replication with Shi et al's original analyses. One is that the achieved sample size is much smaller. The other is that there are some differences to the odds ratios reported by Shi et al for the effect of policy 'liberalization'.

Shi et al report including 172,894 individuals in their analysis. There are over 170,000 respondents in the relevant age category of the original HBSC dataset. But many of them are missing data on variables that are used in these models. In particular, many of them miss data for the number of brothers and number of sisters. These are used to calculate the derived variable for 'number of siblings'. As Shi et al do not report using any imputation techniques to fill in the missing data, these respondents were simply omitted from the replication. This left an achieved sample sizes of 115,504. As Shi et al have not responded to correspondence on this issue, it remains uncertain how they achieved their reported sample size.

Interestingly, the odds ratios calculated for cannabis use by girls in 'liberalized' countries are higher in this replication than in the original analysis by Shi et al. However, in the course of carrying out the replication, it became apparent that there were at least three ways in which the analysis could be improved.

\section{Re-analysis of the HBSC data}

Amendments to the analysis were introduced in order to increase the theoretical relevance and to improve the specification of the models for analysis. They were:

- Inclusion of respondents who missed data on 'number of siblings'.

- Inclusion of data from Sweden from the 2005/6 and 2009/10 HBSC waves.

- Inclusion of random slopes for gender across countries in the regression models.

As can be seen from both Tables 2 and 3, the predictive effect of the 'number of siblings' variable on the odds for adolescent cannabis use was always small and was usually not statistically significant. And, as noted above, including this variable in the 
analysis forced many respondents for whom these data were missing to be omitted. While multilevel models with maximum likelihood estimation provide unbiased estimates when data are missing at random, there is no particular reason to suppose that respondents with missing data on 'number of siblings' were missing at random. For example, there was heterogeneity in the proportion that was missing across countries. Only 2.1 per cent or respondents in Canada missed responses for this variable, compared to 61.1 per cent in Russia. The proportion of respondents who were missing for this variable was also significantly lower among people who did than did not report lifetime cannabis use (15.6 compared to 18.2 per cent. $\chi^{2}=$ 129.4, $\mathrm{p}<0.001$, $d f=1$ ). So it was decided to exclude this variable from the models for re-analysis.

To test hypotheses on the link between policy type and cannabis use, it is preferable to include as many theoretically relevant country cases as possible. Sweden is theoretically an interesting case in drug policy. It has a (contested) reputation for having a low levels of cannabis use because it has a prohibitionist policy (Cohen, 2006; UNODC, 2007). Shi et al did not include data from Sweden from the 2005/6 and 2009/10 waves of the HBSC survey in their models. Data are available from Sweden in those survey years, but the cannabis use questions that were asked were coded slightly differently in the HBSC dataset for Sweden than for other countries. In Sweden in 2005/6 and 2009/10, responses did not include a category that the person had used cannabis '40 times or more'. This means that it is not possible to create a variable for what Shi et al call 'regular' cannabis use for Sweden in those years. But these waves did include comparable data for 'lifetime and 'past year' use. In all countries, the response in the HBSC dataset identifies whether the respondent has 'never' used cannabis in their lifetime or the past year, or has used cannabis at least once in these periods.

Including these Swedish respondents and omitting the 'number of siblings' variable boosted the achieved sample size for the re-analysis to 139,103 . This not only increases the power to observe statistically significant associations between independent and dependent variables. It also reduces the possibility of bias through selective non-response to questions on numbers of siblings and from exclusion of 
most of the Swedish respondents. The choice to improve the analysis in these ways is therefore justified on both analytical and theoretical grounds. However, it means that the re-analysis could not examine 'regular' cannabis use.

Shi et al's analysis suggested that gender is a powerful predictor of adolescent cannabis use, with girls being less likely to report it than boys. If the effect of gender differs between countries, then this may affect the results of the analysis. When there is heterogeneity in the relationship between an independent variable (e.g. gender) and a dependent variable (e.g. cannabis use) between the units at level 2 of the model (e.g. countries), then it is usual to include both the random and the fixed effect of that variable in order to improve goodness of fit of the model to the data (Raudenbush \& Bryk, 2002). Indeed, it has been shown that omission of such 'random slope variation... will tend to increase the risk of Type I error' (Barr, Levy, Scheepers, \& Tily, 2013, p. 263).

There are good reasons to assume that gender differences in cannabis use do vary between countries (Chapman et al., 2017; Ter Bogt et al., 2014). Bivariate analysis of the HBSC data showed that the relationship between gender and cannabis use varied substantially across countries. For example, the unadjusted odds ratio for lifetime cannabis use by boys compared to girls (across all survey waves) was 1.08 (95\% confidence interval: $0.99-1.19)$ in Canada, but it was $2.39(1.88-3.03)$ in Greece.

So the models reported in Table 4 include the random slope for gender across countries. ${ }^{4}$ To look at the effect of policy 'liberalization' on boys, the models were

\footnotetext{
4 The equation of the average fitted regression line for the model with random slopes for gender across countries is: $\log \left(\frac{P_{i j}}{1-P_{i j}}\right)=\beta_{0}+\beta_{1}$ BothParHome $_{i j}+\beta_{2}$ NumFriends $_{i j}+\beta_{3}$ FamSup $_{i j}+\beta_{4}$ FriendSup $_{i j}$ $+\beta_{5}$ TimeFriends $_{i j}+\beta_{6}$ PsychProb $_{i j}+\beta_{7}$ FAS $_{i j}+\beta_{8} F A S 3_{i j}+\beta_{9}$ PolLib $_{i j}$$$
+\beta_{10} \text { PolLib } * \text { Boy }_{i j}+\beta_{11} \text { GDP }_{i j}+\beta_{12} \text { GDP } 3_{i j}+\beta_{13} \text { HBSC2006 }_{i j}
$$$$
+\beta_{14} \text { HBSC2010 } i j+\beta_{15} \text { Gender }_{i j}+u_{0 j}+u_{15 j} \text { Gender }_{i j}+\varepsilon_{i j}
$$ 
also run with the coding for gender reversed (i.e. 0 for boys and 1 for girls). The results of these separate models for the main effect of policy 'liberalization' on boys are shown in a separate row in Table 4. Full results are given in Supplementary Table 2.

Table 4: Multilevel logistic regressions for adolescent cannabis use. Results (odds ratios) of re-analysis of HBSC data, including random slope for gender across countries $(N=139,103)$

\begin{tabular}{|c|c|c|c|c|}
\hline \multirow[b]{2}{*}{ Individual-level variables } & \multicolumn{2}{|l|}{ Lifetime use } & \multicolumn{2}{|c|}{ Past year use } \\
\hline & Odds ratio & Sig' & Odds ratio & Sig' \\
\hline Boy & 2.09 & $* * *$ & 2.08 & $* * *$ \\
\hline Living with both parents & 0.67 & $* * *$ & 0.69 & $* * *$ \\
\hline Family communication & 0.76 & $* * *$ & 0.76 & $* * *$ \\
\hline Friend communication & 1.40 & $* * *$ & 1.39 & $* * *$ \\
\hline Number of friends & 1.01 & $*$ & 1.01 & \\
\hline Time spent with friends & 1.42 & $* * *$ & 1.42 & $* * *$ \\
\hline Psychological complaints & 1.49 & $* * *$ & 1.51 & $* * *$ \\
\hline Family affluence $=$ medium & 1.10 & $* * *$ & 1.12 & $* * *$ \\
\hline Family affluence = high & 1.19 & $* * *$ & 1.23 & $* * *$ \\
\hline \multicolumn{5}{|l|}{ Country-level variables } \\
\hline Cannabis liberalization $^{\dagger}$ & 1.08 & & 1.02 & \\
\hline Cannabis liberalization*boy & 0.87 & $* *$ & 0.94 & \\
\hline Per-capita GDP level = second tertile & 1.19 & & 1.22 & \\
\hline Per-capita GDP level = third tertile & 1.21 & & 1.24 & \\
\hline Survey $=2005 / 6$ & 0.83 & $* * *$ & 0.75 & $* * *$ \\
\hline Survey $=2009 / 10$ & 0.73 & $* * *$ & 0.67 & $* * *$ \\
\hline \multicolumn{5}{|l|}{${ }^{\dagger}$ Effect of cannabis liberalisation for girls } \\
\hline $\begin{array}{l}\text { Effect of cannabis liberalization for } \\
\text { boys }\end{array}$ & 0.94 & & 0.97 & \\
\hline$* p<0.05, * * p<0.01, * * * p<0.001$ & & & & \\
\hline
\end{tabular}

The justification for including random slopes for gender in the models was that this would provide a better analysis of the revised dataset than a model which did not take account of the varying relationship between gender and cannabis use across countries. This assumption was tested by running supplementary models which used the revised dataset, but did not include random slopes for gender (see Supplementary Table 3). The differences between the values for log-likelihood, the

+ GDPS3 + HBSC2006 + HBSC2010 + (1 + Gender | Country), family = binomial("logit"), data = [the dataset $]$, weight $=$ Weight). 
Bayesian Information Criteria (BIC) and the Akaike Information Criteria (AIC) indicate differences in the goodness of fit between these models (Akaike, 1985; Burnham \& Anderson, 2002; Pan, 2001; Schwarz, 1978). As can be seen by comparing these indicators between Supplementary Tables 2 and 3, the analysis which included random slopes for gender did indeed provide better fit to the data than models which did not include this effect.

The main point to arise out of this re-analysis, as seen in Table 4, is: when including more of the available data (including more data from the theoretically interesting country of Sweden) and the varying effect of gender across countries, the association between policy 'liberalization' and lifetime and past year cannabis use becomes statistically non-significant at the five per cent level, for both boys and girls. With these models, it is not possible either to confirm or discard a relationship (in either direction) between policy 'liberalization' and adolescent cannabis use. This suggests that analysis without these amendments risks making the Type I error of falsely rejecting the null hypothesis that policy 'liberalization' is not associated with levels of adolescent cannabis use.

\section{Conclusion}

This article has shown that the approach taken by Shi et al does not provide robust support to their conclusion. The decisions made in the re-analysis reported here (i.e. to include more of the theoretically relevant data and to use better fitting models) are justified on the basis that - both theoretically and analytically - they provide a better test of the hypothesis that Shi et al set out to test. Different model specifications often suggest different conclusions, which reinforces the need for careful consideration and justification of analyses. Future tests of the effect of drug policy on use may support the progress of 'open science' by pre-registration of hypotheses and model specifications, in order to increase transparency and the robustness of hypothesis testing (Humphreys et al., 2013).

This article presents a small step in the direction towards the improved understanding that policy makers and publics need. It cannot demonstrate that there 
is no causal association between policy 'liberalization' and rates of adolescent cannabis use. But it has shown that one piece of evidence that is being used in the debate over prohibition - i.e. the association between policy 'liberalization' and higher odds of adolescent cannabis use that Shi et al reported - does not hold when subject to correct interpretation of Shi et al's numerical results, cannot be replicated precisely from the same data, and is not statistically significant in a re-analysis that improved model fit and uses a larger, more theoretically relevant sample of HBSC respondents.

\section{Acknowledgements}

HBSC is an international study carried out in collaboration with WHO/EURO. The International Coordinator of the 2001/02, 2005/06 and 2009/10 survey was Prof. Candace Currie and the Data Bank Manager was Prof. Oddrun Samdal. The 2001/02, 2005/06 and 2009/10 survey was conducted by Principal Investigators in 38 countries. I am grateful to them all. For details, see http://www.hbsc.org. An earlier version of this article was presented at the International Society for the Study of Drug Policy's Regional Meeting for the Americas in New York, April 2016. I am also grateful for comments received there. Thanks also to anonymous reviewers and to $\mathrm{Dr}$ Jack Cunliffe for their comments on previous versions of this article. The analysis presented here was carried out while the author was on a visiting professorial fellowship at the Drug Policy Modelling Program, UNSW Australia. No funding was received for this research.

\section{References}

Akaike, H. (1985). Prediction and Entropy. In A. C. Atkinson \& S. E. Fienberg (Eds.), A Celebration of Statistics (pp. 1-24). New York: Springer. https://doi.org/10.1007/978-1-4613-8560-8_1

Babor, T., Caulkins, J., Edwards, G., Fischer, B., Foxcroft, D., Humphreys, K., ... Strang, J. (2010). Drug Policy and the Public Good. Oxford: Oxford University Press.

Barr, D. J., Levy, R., Scheepers, C., \& Tily, H. J. (2013). Random effects structure for confirmatory hypothesis testing: Keep it maximal. Journal of Memory and Language, 68(3), 255-278.

Bates, D., Mächler, M., Bolker, B., \& Walker, S. (2015). Fitting Linear Mixed-Effects 
Models Using Ime4. Journal of Statistical Software, 67(1), 1-48.

Burdzovic Andreas, J., \& Bretteville-Jensen, A. L. (2017). Ready, willing, and able: the role of cannabis use opportunities in understanding adolescent cannabis use. Addiction, 112(11), 1973-1982.

Burnham, K. P., \& Anderson, D. R. (2002). Model Selection and Multi- model Inference: A Practical Information-Theoretic Approach. New York: Springer.

Chapman, C., Slade, T., Swift, W., Keyes, K., Tonks, Z., \& Teesson, M. (2017). Evidence for Sex Convergence in Prevalence of Cannabis Use: A Systematic Review and Meta-Regression. Journal of Studies on Alcohol and Drugs, 78(3), 344-352.

Cohen, P. (2006). Looking at the UN, smelling a rat. A comment on 'Sweden's succesful drug policy: a review of the evidence' UNODC September 2006. Amsterdam: CEDRO, University of Amsterdam.

Crowley, D., Collins, C., Delargy, I., Laird, E., \& Van Hout, M. C. (2017). Irish general practitioner attitudes toward decriminalisation and medical use of cannabis: Results from a national survey. Harm Reduction Journal, 14(1), 1-8.

Dirisu, O., Shickle, D., \& Elsey, H. (2016). Influence of legal status on the uptake of cannabis in young people. Current Opinion in Psychiatry, 29(4), 231-235.

HBSC Network. (2014). Health Behaviour is School-Aged Children (HBSC): Terms of Reference. Retrieved June 18, 2015, from http://www.hbsc.org/about/HBSC ToR.pdf

Humphreys, M., de la Sierra, R. S., \& van der Windt, P. (2013). Fishing, Commitment, and Communication: A Proposal for Comprehensive Nonbinding Research Registration. Political Analysis, 21, 1-20.

Hunt, P., Miles, J. N. V., \& Boustead, A. E. (2013). Understanding Patterns in Medical Marijuana Laws: A Latent Class and Transition Analysis. Santa Monica, CA: RAND Corporation.

Mark, K., Gryczynski, J., Axenfeld, E., Schwartz, R. P., \& Terplan, M. (2017). Pregnant Women's Current and Intended Cannabis Use in Relation to Their Views Toward Legalization and Knowledge of Potential Harm. Journal of Addiction Medicine, 11(3), 211-216.

Marsden, J., Eastwood, B., Jones, H., Bradbury, C., Hickman, M., Knight, J., ... the National Drug Treatment Monitoring System Outcomes Study, G. (2012). Risk adjustment of heroin treatment outcomes for comparative performance assessment in England. Addiction, 107(12), 2161-2172.

McLean, K. (2018). A kind of peace: Tracking the reflexive and resilient drug war. International Journal of Drug Policy, 51, 117-120.

Munafò, M. R. (2016). Opening up addiction science. Addiction, 111(3), 387-388.

Munksgaard, R., Demant, J., \& Branwen, G. (2016). A replication and methodological critique of the study "Evaluating drug trafficking on the Tor 
Network." International Journal of Drug Policy.

Murray, R. M., Quigley, H., Quattrone, D., Englund, A., \& Di Forti, M. (2016). Traditional marijuana, high-potency cannabis and synthetic cannabinoids: increasing risk for psychosis. World Psychiatry, 15(3), 195-204.

Open Science Collaboration. (2015). Estimating the reproducibility of psychological science. Science, 349(6521), DOI: 10.1126/science.aac4716.

Pacini, M., Maremmani, A. G. I., Patricio, L., Barra, M., \& Maremmani, I. (2017). Crime issues in Substance Use Disorders: Need for a medically-based algorithm, 19(October), 63-72.

Pan, W. (2001). Akaike's information criterion in generalized estimating equations. Biometrics, 57, 120-125.

Peltzer, K., \& Pengpid, S. (2017). Cannabis and Amphetamine Use Among Adolescents in Five Asian Countries. Central Asian Journal of Global Health, 6(1).

Raudenbush, S. W., \& Bryk, A. S. (2002). Hierarchical linear models: applications and data analysis methods, 2nd edition. Thousand Oaks, CA: Sage.

Roberts, C., Freeman, J., Samdal, O., Schnohr, C. W., Looze, M. E., Nic Gabhainn, S., ... lannotti, R. (2009). The Health Behaviour in School-aged Children (HBSC) study: Methodological developments and current tensions. International Journal of Public Health, 54(SUPPL. 2), 140-150.

Røgeberg, O., \& Stevens, A. (2016). Liberalization and adolescent cannabis use issues with interpretation and country coding. Retrieved January 22, 2016, from http://www. plosone.org/annotation/listThread.action?root=87919

Room, R., Fischer, B., Hall, W., Lenton, S., Reuter, P., \& Fielding, A. (2008). Cannabis Policy: Moving Beyond Stalemate. The Global Cannabis Comission Report. Oxford: Beckley Foundation.

Schwarz, G. (1978). Estimating the dimension of a model. Annals of Statistics, 6, 461-464.

Shi, Y., Lenzi, M., \& An, R. (2015). Cannabis Liberalization and Adolescent Cannabis Use : A Cross-National Study in 38 Countries. PLoS ONE, 10(11), e0143562. https://doi.org/10.1371/journal.pone.0143562

Silberzahn, R., \& Uhlmann, E. L. (2015). Crowdsourced research: Many hands make tight work. Nature, 526(7572), 189-191.

Ter Bogt, T. F. M., de Looze, M., Molcho, M., Godeau, E., Hublet, A., Kokkevi, A., ... Pickett, W. (2014). Do societal wealth, family affluence and gender account for trends in adolescent cannabis use? A 30 country cross-national study. Addiction, 109, 273-283.

Turan Sonmez, F., Kilicasla, O., \& Akbas, T. (2017). Medicine Cannabis Use in Adolescence: A 4-Year Experience, 6(1), 683-685. 
UNODC. (2007). Sweden's Successful Drug Policy: A Review of the Evidence. Vienna: United Nations Office on Drugs and Crime.

Waddell K, \& Wilson MG. (2017). Rapid Synthesis: Examining the Impact of Decriminalizing or Legalizing Cannabis for Recreational Use. Hamilton, Canada: McMaster Health Forum/Michael G. DeGroote Centre for Medicinal Cannabis Research.

Wall, M. M., Mauro, C., Hasin, D. S., Keyes, K. M., Cerda, M., Martins, S. S., \& Feng, T. (2016). Prevalence of marijuana use does not differentially increase among youth after states pass medical marijuana laws: Commentary on Stolzenberg et al. (2015) and reanalysis of US National Survey on Drug Use in Households data 2002-2011. International Journal of Drug Policy, 29, 9-13.

Weinberger, A. H., Pacek, L. R., Wall, M. M., Zvolensky, M. J., Copeland, J., Galea, S., ... Goodwin, R. D. (2018). Trends in cannabis use disorder by cigarette smoking status in the United States, 2002 to 2016. Drug and Alcohol Dependence, Onine early. https://doi.org/10.1016/j.drugalcdep.2018.06.016 


\section{Supplementary Tables}

N.B. Estimates reported in these tables relate to log odds, not odds ratios.

\begin{tabular}{|c|c|c|c|c|c|c|}
\hline \multicolumn{7}{|c|}{$\begin{array}{l}\text { Supplementary Table 1: Multi-level logistic regressions for adolescent cannabis use. Results } \\
\text { of replication of Shi et al } 2015(N=115,504) \text {. }\end{array}$} \\
\hline \multirow[b]{2}{*}{ Random effect } & \multicolumn{2}{|c|}{ Lifetime use } & & & & \\
\hline & Variance & S.D. & & & & \\
\hline Country (intercept) & 0.602 & 0.776 & \multirow[b]{2}{*}{$\underline{z \text { value }}$} & \multirow[b]{2}{*}{$\underline{\operatorname{Pr}(>|z|)}$} & & \\
\hline$\underline{\text { Fixed Effects }}$ & Estimate & $\underline{\text { S.E. }}$ & & & \multicolumn{2}{|c|}{ 95\% confidence interval } \\
\hline Intercept & -4.429 & 0.213 & -20.83 & $<0.001$ & -4.846 & -4.012 \\
\hline Boy & 0.692 & 0.025 & 27.91 & $<0.001$ & 0.643 & 0.740 \\
\hline Living with both parents & -0.413 & 0.019 & -22.31 & $<0.001$ & -0.450 & -0.377 \\
\hline Number of siblings & -0.005 & 0.006 & -0.81 & 0.419 & -0.016 & 0.007 \\
\hline Family communication & -0.280 & 0.010 & -27.86 & $<0.001$ & -0.299 & -0.260 \\
\hline Friend communication & 0.356 & 0.013 & 28.15 & $<0.001$ & 0.331 & 0.381 \\
\hline Number of friends & 0.016 & 0.006 & 2.57 & 0.010 & 0.004 & 0.028 \\
\hline Time spent with friends & 0.354 & 0.005 & 66.76 & $<0.001$ & 0.344 & 0.365 \\
\hline Psychological complaints & 0.404 & 0.009 & 42.57 & $<0.001$ & 0.385 & 0.422 \\
\hline Family affluence $=$ medium & 0.116 & 0.025 & 4.69 & $<0.001$ & 0.068 & 0.165 \\
\hline Family affluence = high & 0.197 & 0.025 & 7.96 & $<0.001$ & 0.149 & 0.246 \\
\hline Cannabis liberalization & 0.286 & 0.039 & 7.35 & $<0.001$ & 0.209 & 0.362 \\
\hline Cannabis liberalization*boy & -0.244 & 0.033 & -7.51 & $<0.001$ & -0.308 & -0.181 \\
\hline Per-capita GDP level = 2nd tertile & 0.273 & 0.311 & 0.88 & 0.380 & -0.339 & 0.885 \\
\hline Per-capita GDP level = 3rd tertile & 0.492 & 0.303 & 1.62 & 0.104 & -0.100 & 1.084 \\
\hline Survey $=2005 / 6$ & -0.272 & 0.023 & -12.03 & $<0.001$ & -0.316 & -0.228 \\
\hline Survey $=2009 / 10$ & -0.350 & 0.023 & -15.28 & $<0.001$ & -0.395 & -0.305 \\
\hline \multicolumn{3}{|c|}{ Past year use } & & & & \\
\hline$\underline{\text { Random effect }}$ & Variance & $\underline{\text { S.D. }}$ & & & & \\
\hline Country (intercept) & 0.540 & 0.735 & & & & \\
\hline Fixed Effects & Estimate & S.E. & $\underline{z \text { value }}$ & $\operatorname{Pr}(>|z|)$ & \multicolumn{2}{|c|}{ 95\% confidence interval } \\
\hline Intercept & -4.728 & 0.205 & -23.11 & $<0.001$ & -5.129 & -4.327 \\
\hline Boy & 0.661 & 0.027 & 24.45 & $<0.001$ & 0.608 & 0.714 \\
\hline Living with both parents & -0.377 & 0.020 & -18.90 & $<0.001$ & -0.416 & -0.338 \\
\hline Number of siblings & -0.003 & 0.006 & -0.40 & 0.689 & -0.015 & 0.010 \\
\hline Family communication & -0.281 & 0.011 & -26.14 & $<0.001$ & -0.302 & -0.260 \\
\hline Friend communication & 0.355 & 0.014 & 25.89 & $<0.001$ & 0.329 & 0.382 \\
\hline Number of friends & 0.010 & 0.007 & 1.44 & 0.149 & -0.004 & 0.023 \\
\hline Time spent with friends & 0.351 & 0.006 & 61.74 & $<0.001$ & 0.340 & 0.362 \\
\hline Psychological complaints & 0.415 & 0.010 & 41.01 & $<0.001$ & 0.395 & 0.434 \\
\hline Family affluence $=$ medium & 0.131 & 0.027 & 4.84 & $<0.001$ & 0.078 & 0.183 \\
\hline Family affluence $=$ high & 0.225 & 0.027 & 8.37 & $<0.001$ & 0.172 & 0.278 \\
\hline Cannabis liberalization & 0.216 & 0.042 & 5.11 & $<0.001$ & 0.133 & 0.298 \\
\hline Cannabis liberalization*boy & -0.202 & 0.035 & -5.72 & $<0.001$ & -0.271 & -0.133 \\
\hline Per-capita GDP level = 2nd tertile & 0.379 & 0.296 & 1.28 & 0.201 & -0.202 & 0.959 \\
\hline Per-capita GDP level = 3rd tertile & 0.602 & 0.287 & 2.10 & 0.036 & 0.040 & 1.164 \\
\hline Survey $=2005 / 6$ & -0.382 & 0.024 & -15.70 & $<0.001$ & -0.429 & -0.334 \\
\hline Survey $=2009 / 10$ & -0.429 & 0.025 & -17.50 & $<0.001$ & -0.477 & -0.381 \\
\hline
\end{tabular}




\begin{tabular}{|c|c|c|c|c|c|c|}
\hline \multicolumn{7}{|c|}{$\begin{array}{l}\text { Supplementary Table } 1 \text { (continued): Multi-level logistic regressions for adolescent cannabis } \\
\text { use. Results of replication of Shi et al } 2015(N=115,504) \text {. }\end{array}$} \\
\hline \multirow[b]{2}{*}{$\underline{\text { Random effect }}$} & \multicolumn{2}{|c|}{ Regular use } & & & & \\
\hline & Variance & $\underline{\text { S.D. }}$ & & & & \\
\hline Country (intercept) & 0.714 & 0.845 & & & & \\
\hline Fixed Effects & Estimate & $\underline{\text { S.E. }}$ & $\underline{z \text { value }}$ & $\underline{\operatorname{Pr}(>|z|)}$ & $\underline{95 \% \text { confi }}$ & ce interval \\
\hline Intercept & -7.292 & 0.270 & -27.03 & $<0.001$ & -7.821 & -6.763 \\
\hline Boy & 1.113 & 0.060 & 18.69 & $<0.001$ & 0.996 & 1.230 \\
\hline Living with both parents & -0.465 & 0.038 & -12.40 & $<0.001$ & -0.539 & -0.392 \\
\hline Number of siblings & 0.033 & 0.011 & 3.12 & 0.002 & 0.012 & 0.054 \\
\hline Family communication & -0.281 & 0.020 & -14.06 & $<0.001$ & -0.321 & -0.242 \\
\hline Friend communication & 0.240 & 0.026 & 9.27 & $<0.001$ & 0.189 & 0.291 \\
\hline Number of friends & -0.009 & 0.014 & -0.62 & 0.535 & -0.036 & 0.018 \\
\hline Time spent with friends & 0.465 & 0.011 & 42.21 & $<0.001$ & 0.444 & 0.487 \\
\hline Psychological complaints & 0.488 & 0.019 & 26.11 & $<0.001$ & 0.451 & 0.524 \\
\hline Family affluence $=$ medium & 0.000 & 0.052 & 0.00 & 0.999 & -0.103 & 0.102 \\
\hline Family affluence = high & -0.002 & 0.052 & -0.04 & 0.966 & -0.104 & 0.099 \\
\hline Cannabis liberalization & 0.258 & 0.089 & 2.89 & 0.004 & 0.083 & 0.433 \\
\hline Cannabis liberalization*boy & -0.238 & 0.074 & -3.23 & 0.001 & -0.383 & -0.094 \\
\hline Per-capita GDP level = 2nd tertile & 0.990 & 0.353 & 2.81 & 0.005 & 0.299 & 1.681 \\
\hline Per-capita GDP level = 3rd tertile & 1.345 & 0.340 & 3.95 & $<0.001$ & 0.678 & 2.013 \\
\hline Survey $=2005 / 6$ & -0.369 & 0.047 & -7.85 & $<0.001$ & -0.461 & -0.277 \\
\hline Survey $=2009 / 10$ & -0.537 & 0.048 & -11.28 & $<0.001$ & -0.630 & -0.444 \\
\hline
\end{tabular}


Supplemetary Table 2: Multi-level logistic regressions for adolescent cannabis use. Including random slope for gender across countries $(N=139,103)$.

\begin{tabular}{|c|c|c|c|c|c|c|}
\hline & Lifetime use & & & & & \\
\hline$\underline{\text { Random effect }}$ & $\underline{\text { Variance }}$ & $\underline{\text { S.D. }}$ & Correlation & & & \\
\hline Country (intercept) & 0.762 & 0.873 & & & & \\
\hline Gender & 0.055 & 0.235 & -0.65 & & & \\
\hline Fixed Effects & Estimate & S.E. & $\underline{z \text { value }}$ & $\underline{\operatorname{Pr}(>|z|)}$ & \multicolumn{2}{|c|}{ 95\% confidence interval } \\
\hline Intercept & -4.187 & 0.221 & -18.93 & $<0.001$ & -4.620 & -3.753 \\
\hline Boy & 0.735 & 0.048 & 15.17 & $<0.001$ & 0.640 & 0.831 \\
\hline Living with both parents & -0.405 & 0.017 & -23.88 & $<0.001$ & -0.439 & -0.372 \\
\hline Family communication & -0.276 & 0.009 & -30.05 & $<0.001$ & -0.294 & -0.258 \\
\hline Friend communication & 0.336 & 0.012 & 29.16 & $<0.001$ & 0.314 & 0.359 \\
\hline Number of friends & 0.015 & 0.006 & 2.55 & 0.011 & 0.003 & 0.026 \\
\hline Time spent with friends & 0.353 & 0.005 & 72.42 & $<0.001$ & 0.343 & 0.362 \\
\hline Psychological complaints & 0.398 & 0.009 & 45.96 & $<0.001$ & 0.381 & 0.415 \\
\hline Family affluence = medium & 0.098 & 0.023 & 4.34 & $<0.001$ & 0.054 & 0.142 \\
\hline Family affluence = high & 0.177 & 0.023 & 7.82 & $<0.001$ & 0.133 & 0.221 \\
\hline Cannabis liberalization & 0.074 & 0.040 & 1.84 & 0.066 & -0.005 & 0.153 \\
\hline Cannabis liberalization*boy & -0.139 & 0.049 & -2.85 & 0.004 & -0.235 & -0.043 \\
\hline Per-capita GDP level = 2nd tertile & 0.170 & 0.297 & 0.57 & 0.566 & -0.412 & 0.752 \\
\hline Per-capita GDP level = 3rd tertile & 0.190 & 0.304 & 0.62 & 0.532 & -0.407 & 0.787 \\
\hline Survey = 2005/6 & -0.185 & 0.020 & -9.04 & $<0.001$ & -0.225 & -0.145 \\
\hline Survey $=2009 / 10$ & -0.314 & 0.021 & -15.24 & $<0.001$ & -0.354 & -0.274 \\
\hline $\begin{array}{l}\text { Effect of cannabis liberalisation for } \\
\text { boys }\end{array}$ & -0.065 & 0.038 & -1.70 & 0.088 & -0.139 & -0.010 \\
\hline Log-likelihod & -57502.0 & & & & & \\
\hline AIC & 115042.1 & & & & & \\
\hline \multirow[t]{2}{*}{$\mathrm{BIC}$} & 115229.1 & & & & & \\
\hline & \multicolumn{2}{|l|}{ Past Year use } & & & & \\
\hline Random effect & Variance & $\underline{\text { S.D. }}$ & Correlation & & & \\
\hline Country (intercept) & 0.766 & 0.875 & & & & \\
\hline Gender & 0.073 & 0.271 & -0.79 & & & \\
\hline Fixed Effects & Estimate & S.E. & $\underline{z \text { value }}$ & $\underline{\operatorname{Pr}(>|z|)}$ & \multicolumn{2}{|c|}{ 95\% confidence interval } \\
\hline Intercept & -4.419 & 0.207 & -21.37 & $<0.001$ & -4.824 & -4.014 \\
\hline Boy & 0.733 & 0.055 & 13.45 & $<0.001$ & 0.626 & 0.840 \\
\hline Living with both parents & -0.377 & 0.018 & -20.71 & $<0.001$ & -0.413 & -0.342 \\
\hline Family communication & -0.275 & 0.010 & -27.98 & $<0.001$ & -0.294 & -0.256 \\
\hline Friend communication & 0.329 & 0.012 & 26.41 & $<0.001$ & 0.305 & 0.354 \\
\hline Number of friends & 0.008 & 0.006 & 1.24 & 0.213 & -0.004 & 0.020 \\
\hline Time spent with friends & 0.348 & 0.005 & 66.74 & $<0.001$ & 0.338 & 0.358 \\
\hline Psychological complaints & 0.410 & 0.009 & 44.42 & $<0.001$ & 0.392 & 0.428 \\
\hline Family affluence = medium & 0.109 & 0.025 & 4.44 & $<0.001$ & 0.061 & 0.157 \\
\hline Family affluence = high & 0.205 & 0.025 & 8.35 & $<0.001$ & 0.157 & 0.253 \\
\hline Cannabis liberalization & 0.022 & 0.044 & 0.51 & 0.611 & -0.064 & 0.109 \\
\hline Cannabis liberalization*boy & -0.057 & 0.052 & -1.09 & 0.277 & -0.159 & 0.046 \\
\hline Per-capita GDP level = 2nd tertile & 0.201 & 0.254 & 0.79 & 0.430 & -0.297 & 0.699 \\
\hline Per-capita GDP level = 3rd tertile & 0.212 & 0.255 & 0.83 & 0.405 & -0.287 & 0.711 \\
\hline Survey $=2005 / 6$ & -0.283 & 0.022 & -12.89 & $<0.001$ & -0.326 & -0.240 \\
\hline Survey $=2009 / 10$ & -0.400 & 0.022 & -18.14 & $<0.001$ & -0.444 & -0.357 \\
\hline $\begin{array}{l}\text { Effect of cannabis liberalisation for } \\
\text { boys }\end{array}$ & -0.034 & 0.041 & -0.85 & 0.397 & -0.114 & 0.045 \\
\hline Log-likelihod & -50901.6 & & & & & \\
\hline AIC & 101841.2 & & & & & \\
\hline $\mathrm{BIC}$ & 102028.2 & & & & & \\
\hline
\end{tabular}




\begin{tabular}{|c|c|c|c|c|c|c|}
\hline \multicolumn{7}{|c|}{$\begin{array}{l}\text { Supplemetary Table 3: Multi-level logistic regressions for adolescent cannabis use. Revised } \\
\text { dataset. No random slope for gender across countries }(N=139,103) \text {. }\end{array}$} \\
\hline \multirow[b]{2}{*}{$\underline{\text { Random effect }}$} & \multicolumn{2}{|c|}{ Lifetime use } & \multirow[b]{4}{*}{$\underline{z \text { value }}$} & \multirow[b]{4}{*}{$\underline{\operatorname{Pr}(>|z|)}$} & & \\
\hline & $\underline{\text { Variance }}$ & $\underline{\text { S.D. }}$ & & & & \\
\hline Country (intercept) & 0.600 & 0.775 & & & & \\
\hline Fixed Effects & Estimate & S.E. & & & \multicolumn{2}{|c|}{$95 \%$ confidence interval } \\
\hline Intercept & -4.364 & 0.209 & -20.85 & $<0.001$ & -4.774 & -3.954 \\
\hline Boy & 0.687 & 0.023 & 30.50 & $<0.001$ & 0.643 & 0.731 \\
\hline Living with both parents & -0.404 & 0.017 & -23.84 & $<0.001$ & -0.438 & -0.371 \\
\hline Family communication & -0.277 & 0.009 & -30.14 & $<0.001$ & -0.295 & -0.259 \\
\hline Friend communication & 0.338 & 0.012 & 29.31 & $<0.001$ & 0.315 & 0.361 \\
\hline Number of friends & 0.017 & 0.006 & 3.00 & $<0.001$ & 0.006 & 0.028 \\
\hline Time spent with friends & 0.352 & 0.005 & 72.34 & $<0.001$ & 0.342 & 0.361 \\
\hline Psychological complaints & 0.396 & 0.009 & 45.83 & $<0.001$ & 0.379 & 0.413 \\
\hline Family affluence $=$ medium & 0.103 & 0.023 & 4.59 & $<0.001$ & 0.059 & 0.147 \\
\hline Family affluence = high & 0.181 & 0.023 & 8.00 & $<0.001$ & 0.137 & 0.225 \\
\hline Cannabis liberalization & 0.119 & 0.034 & 3.46 & $<0.001$ & 0.052 & 0.186 \\
\hline Cannabis liberalization*boy & -0.228 & 0.030 & -7.63 & $<0.001$ & -0.286 & -0.169 \\
\hline Per-capita GDP level = 2nd tertile & 0.427 & 0.310 & 1.38 & 0.17 & -0.180 & 1.034 \\
\hline Per-capita GDP level = 3rd tertile & 0.571 & 0.301 & 1.90 & 0.06 & -0.018 & 1.161 \\
\hline Survey $=2005 / 6$ & -0.186 & 0.020 & -9.13 & $<0.001$ & -0.226 & -0.146 \\
\hline Survey = 2009/10 & -0.315 & 0.021 & -15.29 & $<0.001$ & -0.355 & -0.274 \\
\hline Log-likelihod & -57586.2 & & & & & \\
\hline AIC & 115206.4 & & & & & \\
\hline \multirow[t]{2}{*}{$\mathrm{BIC}$} & 115373.7 & & & & & \\
\hline & \multicolumn{2}{|c|}{ Past Year use } & & & & \\
\hline$\underline{\text { Random effect }}$ & $\underline{\text { Variance }}$ & S.D. & & & & \\
\hline Country (intercept) & 0.5347 & 0.7312 & & & & \\
\hline Fixed Effects & Estimate & $\underline{\text { S.E. }}$ & $\underline{z \text { value }}$ & $\underline{\operatorname{Pr}(>|z|)}$ & \multicolumn{2}{|c|}{$\underline{95 \% \text { confidence interva }}$} \\
\hline Intercept & -4.619 & 0.202 & -22.92 & $<0.001$ & -5.014 & -4.224 \\
\hline Boy & 0.659 & 0.025 & 26.84 & $<0.001$ & 0.610 & 0.707 \\
\hline Living with both parents & -0.377 & 0.018 & -20.68 & $<0.001$ & -0.412 & -0.341 \\
\hline Family communication & -0.276 & 0.010 & -28.05 & $<0.001$ & -0.295 & -0.256 \\
\hline Friend communication & 0.331 & 0.012 & 26.55 & $<0.001$ & 0.307 & 0.355 \\
\hline Number of friends & 0.010 & 0.006 & 1.65 & 0.100 & -0.002 & 0.022 \\
\hline Time spent with friends & 0.347 & 0.005 & 66.68 & $<0.001$ & 0.337 & 0.357 \\
\hline Psychological complaints & 0.409 & 0.009 & 44.35 & $<0.001$ & 0.390 & 0.427 \\
\hline Family affluence $=$ medium & 0.114 & 0.024 & 4.68 & $<0.001$ & 0.066 & 0.162 \\
\hline Family affluence $=$ high & 0.209 & 0.024 & 8.53 & $<0.001$ & 0.161 & 0.257 \\
\hline Cannabis liberalization & 0.089 & 0.037 & 2.37 & 0.018 & 0.016 & 0.162 \\
\hline Cannabis liberalization*boy & -0.187 & 0.032 & -5.81 & $<0.001$ & -0.250 & -0.124 \\
\hline Per-capita GDP level = 2nd tertile & 0.522 & 0.294 & 1.78 & 0.076 & -0.054 & 1.098 \\
\hline Per-capita GDP level = 3rd tertile & 0.654 & 0.286 & 2.29 & 0.022 & 0.095 & 1.214 \\
\hline Survey = 2005/6 & -0.285 & 0.022 & -12.97 & $<0.001$ & -0.328 & -0.242 \\
\hline Survey = 2009/10 & -0.400 & 0.022 & -18.15 & $<0.001$ & -0.444 & -0.357 \\
\hline Log-likelihod & -50995.0 & & & & & \\
\hline AIC & 102024.1 & & & & & \\
\hline $\mathrm{BIC}$ & 102191.4 & & & & & \\
\hline
\end{tabular}

